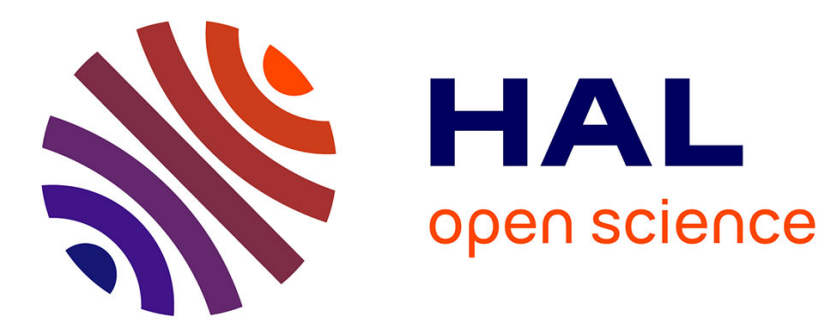

\title{
4 - d expansion for Anderson localization in a strong spin-orbit coupling case \\ S. Hikami
}

\section{To cite this version:}

S. Hikami. 4 - d expansion for Anderson localization in a strong spin-orbit coupling case. Journal de Physique Lettres, 1985, 46 (16), pp.719-722. 10.1051/jphyslet:019850046016071900 . jpa-00232889

\section{HAL Id: jpa-00232889 https://hal.science/jpa-00232889}

Submitted on 1 Jan 1985

HAL is a multi-disciplinary open access archive for the deposit and dissemination of scientific research documents, whether they are published or not. The documents may come from teaching and research institutions in France or abroad, or from public or private research centers.
L'archive ouverte pluridisciplinaire HAL, est destinée au dépôt et à la diffusion de documents scientifiques de niveau recherche, publiés ou non, émanant des établissements d'enseignement et de recherche français ou étrangers, des laboratoires publics ou privés. 


\title{
LE JOURNAL DE PHYSIQUE-LETTRES
}

J. Physique Lett. 46 (1985) L-719 - L-722

15 AOÛT 1985, PAGE L-719

Classification

Physics Abstracts

$71.30-71.55 \mathrm{~J}$

\section{$4-d$ expansion for Anderson localization in a strong spin-orbit coupling case}

\author{
S. Hikami \\ Service de Physique Théorique, CEN-Saclay, 91191 Gif-sur-Yvette Cedex, France
}

(Reçu le 25 avril 1985, accepté le 20 juin 1985)

\begin{abstract}
Résumé. - Le problème de la localisation d'Anderson en présence de fort couplage spin-orbite est étudié dans le développement en $\varepsilon=4-d$ d'un modèle matriciel invariant de jauge. Un point fixe stable dans l'infrarouge est présent et ce modèle appartient à la même classe d'universalité que le modèle $\sigma$ non linéaire. Une expression pour l'exposant de la conductivité est obtenue au premier ordre en $\varepsilon$.
\end{abstract}

\begin{abstract}
The problem of Anderson localization in a strong spin-orbit coupling case is studied by using a gauge invariant matrix model in $\varepsilon=4-d$ expansion. An infrared stable fixed point is present and this model belongs to the same universal class as a nonlinear $\sigma$ model. An expression for the critical exponent of conductivity is obtained in the first order of the $\varepsilon$-expansion.
\end{abstract}

Recently Anderson localization has been investigated in $2+\varepsilon$ dimensions for three cases; the orthogonal, unitary, and symplectic cases [1-4]. The orthogonal case corresponds to the case with no spin-effect. Strong spin-spin interaction or strong magnetic field case belongs to the unitary case. The symplectic case is obtained by a strong spin-orbit interaction [5]. By an $\varepsilon=d-2$ expansion, the critical point for the metal-insulator transition is obtained for the orthogonal case and for the unitary case. However, for the symplectic case, the critical behaviour has not been discussed since, up to four-loop order, the renormalization group $\beta$-function is positive [3].

The Effective Hamiltonian for these three cases are non-compact nonlinear $\sigma$-models. As for usual critical phenomena, it is useful to consider also the $\varepsilon=4-d$ expansion for the strong spinorbit case based on a gauge invariant matrix model. The field-theoretical model which we consider in this letter near four dimensions has already been discussed as a model which belongs to the same universal class as the Grassmannian nonlinear $\sigma$ model and indeed within the $1 / N$ expansion, the equivalence of the two models has been verified [6,7]. The expression of the exponent $v$ of the nonlinear $\sigma$ model agrees with the expression of the gauge invariant matrix model in an 
overlapping large $N$ and small $\varepsilon=4-d$ region.'The Hamiltonian of the gauge invariant matrix model is given by

$$
\begin{aligned}
H=\frac{1}{2}\left[\partial_{\mu} \Phi_{i}^{\alpha}+g A_{\mu i j} \Phi_{j}^{\alpha}\right]^{2} & +\frac{\lambda}{8} \sum_{i, j=1}^{p} \sum_{\alpha=1}^{N} \Phi_{i}^{\alpha} \Phi_{i}^{\alpha} \Phi_{j}^{\alpha} \Phi_{j}^{\alpha} \\
& +\frac{v}{4} \sum_{i . j=1}^{p} \sum_{\alpha \neq \beta} \Phi_{i}^{\alpha} \Phi_{j}^{\alpha} \Phi_{j}^{\beta} \Phi_{i}^{\beta}+\frac{\rho}{8} \sum_{i . j=1}^{p} \sum_{\alpha \neq \beta} \Phi_{i}^{\alpha} \Phi_{i}^{\alpha} \Phi_{j}^{\beta} \Phi_{j}^{\beta} \\
& +\frac{1}{4}\left(\partial_{\mu} A_{v}^{a}-\partial_{v} A_{\mu}^{a}+g C_{a b c} A_{\mu}^{b} A_{v}^{c}\right)^{2}
\end{aligned}
$$

where $\Phi_{i}^{\alpha}(i=1, \ldots, p ; \alpha=1, \ldots, N)$ is $p \times N$ real matrix field, and $A_{\mu}$ is a non-Abelian gauge field belonging to $\mathrm{O}(p)$. We have considered a model in which $\lambda=\rho+2 v$ where a global $\mathrm{O}(N)$ symmetry is present. If this model has an infrared stable fixed point, where bare coupling constants become infinite, the model is reduced to the $\mathrm{O}(N) / \mathrm{O}(p) \times \mathrm{O}(N-p)$ Grassmannian nonlinear $\sigma$ model which has the following Hamiltonian [8]

$$
H=\frac{1}{2 t}\left\{\left[\partial_{\mu} \Phi_{i}^{\alpha}\right]\left[\partial_{\mu} \Phi_{i}^{\alpha}\right]+\left[\Phi_{i}^{\alpha} \partial_{\mu} \Phi_{j}^{\alpha}\right]\left[\Phi_{j}^{\beta} \partial_{\mu} \Phi_{i}^{\beta}\right]\right\}
$$

with

$$
\sum_{\alpha=1}^{N} \Phi_{i}^{\alpha} \Phi_{j}^{\alpha}=\delta_{i j}
$$

The renormalization group $\beta$-functions of $(1)$ are $(\varepsilon=4-d)$

$$
\begin{aligned}
& \beta_{\lambda}=-\varepsilon \lambda+\frac{1}{16 \pi^{2}} \times \\
& \times\left\{(p N+8) \lambda^{2}-4(N-1)(p-1) \lambda v+4(p-1)(N-1) v^{2}-3(p-1) g^{2} \lambda+\frac{3}{4}(p-1) g^{4}\right\} \\
& \beta_{v}=-\varepsilon v+\frac{1}{16 \pi^{2}}\left\{(2 N+2 p-16) v^{2}+12 \lambda v-3(p-1) g^{2} v+\frac{3}{8}(p-2) g^{4}\right\} \\
& \beta_{g}=-\frac{\varepsilon}{2} g-\frac{1}{16 \pi^{2}}\left\{\frac{11}{3}(p-2)-\frac{N}{12}\right\} g^{3} .
\end{aligned}
$$

These $\beta$-functions have infrared stable fixed points for certain domains of the number of components $p$ and $N$. In the large $N$ limit for a fixed $p$, we have

$$
\begin{aligned}
& g_{\mathrm{c}}^{2}=96 \pi^{2} \varepsilon / N \\
& \lambda_{\mathrm{c}}=\frac{16 \pi^{2} \varepsilon}{N}\left(1-\frac{10 p}{N}+\frac{2}{N}\right)+\mathcal{O}\left(\frac{\varepsilon}{N^{3}}\right) \\
& v_{\mathrm{c}}=\frac{8 \pi^{2} \varepsilon}{N}\left(1-\frac{10 p}{N}+\frac{32}{N}\right)+\mathcal{O}\left(\frac{\varepsilon}{N^{3}}\right) .
\end{aligned}
$$

This fixed point is stable for all directions and the critical exponent $v$ agrees with the value of the nonlinear $\sigma$ model in the large $N$ and small $\varepsilon$ limit. This fact shows that Hamiltonian (1) belongs to the same universal class as Hamiltonian (2). 
The Anderson localizations are described by a non-compact nonlinear $\sigma$ model. For the orthogonal case, the nonlinear $\sigma$ model has $\mathrm{O}(p, N-p) / \mathrm{O}(p) \times \mathrm{O}(N-p)$ symmetry with $p=N=0$. For the symplectic case, the symmetry becomes $S_{p}(p, N-p) / S_{p}(p) \times S_{p}(N-p)$ with $p=N=0$. There is an isomorphic relation between the orthogonal group and the symplectic group in the following

$$
\mathrm{O}(-2 N) \simeq S_{p}(N)
$$

and the non-compact case $\beta$-function is obtained from the compact case by changing a sign of the coupling $[3,9,10]$ constant. Therefore, the strong spin-orbit case is described by the compact $\mathrm{O}(N) / \mathrm{O}(p) \times \mathrm{O}(N-p)$ nonlinear $\sigma$ model with $p=N=0$. If the Hamiltonian of (1) has an infrared stable fixed point for $p=N=0$, then the critical behaviour represents the localization transition with a strong spin-orbit case. For $p=N=0$, we have four different points. Two of them are reported here for the first time. They are $(\tilde{\lambda}, \tilde{v})=(0.10814,0.01037),(0.114,0.028)$ $(-0.005,-0.040),(-0.0042,-0.0045)$ where $\lambda=\lambda / 16 \pi^{2} \varepsilon$ and $\tilde{v}=v / 16 \pi^{2} \varepsilon$. The fixed point of the gauge coupling becomes $g_{\mathrm{c}}^{2}=48 \varepsilon \pi^{2} / 44$. By calculating the derivatives of $\beta$-functions, we find that the first fixed point is stable for all directions and the critical exponant $v$ is obtained for general $p$ and $N$.

$$
\begin{aligned}
\frac{1}{v} & =2+\eta_{\Phi 2}-\eta_{\Phi} \\
\eta_{\Phi} & =\beta_{g} \frac{\partial}{\partial g} \ln Z_{\Phi}=\frac{g(p-1)}{44(p-2)-N} \varepsilon \\
\eta_{\Phi^{2}} & =-\frac{p N+2}{16 \pi^{2}} \lambda_{\mathrm{c}}+\frac{(p-1)(N-1)}{8 \pi^{2}} v_{\mathrm{c}}
\end{aligned}
$$

For $p=N=0$, we have

$$
\frac{1}{v}=2-0.297 \varepsilon+\mathcal{O}\left(\varepsilon^{2}\right)
$$

or

$$
v=\frac{1}{2}+0.0742 \varepsilon+\mathcal{O}\left(\varepsilon^{2}\right) .
$$

Stable fixed points are limited to some domains in the $(p, N)$ plane. For the $p=0$ case, we have two regions of stability (i) $8<N<\infty$ and (ii) $-88<N<1.2$. For the $N=0$ case, we have a region of stability as $10-\infty<p<0.5$.

We now comment about the symmetry of $p \leftrightarrow N-p$. For the case of $N=2 p$ (including $p=N=0$ case), we have a manifest $p \leftrightarrow N-p$ symmetry which the Grassmannian nonlinear $\sigma$ model (2) should preserve. However, in the case of $N \neq 2 p$, Hamiltonian (1) has no manifest $p \leftrightarrow N-p$ symmetry and we have not recovered this symmetry even at the fixed point due to the small size of the stability domain in the $(p, N)$ plane. It is possible to write a Hamiltonian of manifest $p \leftrightarrow N-p$ symmetry with two independent $\mathrm{O}(p)$ and $\mathrm{O}(N-p)$ gauge fields. However, the renormalization group calculation does not give us a stable fixed point for all coupling constants except for the $N=2 p$ case.

If there exists a second order phase transition, the conductivity $\sigma$ has the following behaviour near a mobility edge

$$
\sigma=C\left|E-E_{\mathrm{c}}\right|^{\mathrm{s}}
$$

where $C$ is a constant and the exponent of the conductivity $s$ is related to the previous exponent $v$ as

$$
s=(d-2) v .
$$


The symplectic case gives a positive $\beta$-function in two dimensions. Analogous behaviour for the $\beta$-function is obtained in the $N$-vector model $(p=1)$ when we put $N=1$ which corresponds to the Ising model. It is known that Ising model has a lower critical dimensionality at $d=1$. It is possible that the symplectic case has a lower critical dimensionality less than two and possibly one. Therefore, the critical exponent $s$ in two dimensions is considered to be zero from (9) since the exponent $v$ is finite. The assumption of finiteness for the exponent $v$ in two dimensions is also consistent with the small correction of order $\varepsilon$ as (7). The conductivity vanishes at the mobility edge discontinuously in two dimensions since the exponent $s$ is vanishing. In a magneto-resistance experiment, the spin-orbit effect has been studied [11]. It is also interesting to compare the result of (7) with the experimental observations in doped semiconductors [12]. By putting $\varepsilon=1$ in (7) we have a conductivity exponent $s=0.57$. Like ordinary critical phenomena, the mean field behaviour for the conductivity may be present. In our case, we take a mean field value of $v$ as $1 / 2$ and the conductivity exponent $s$ is 1 since we put $d=4$ and $v=1 / 2$ in (9). Therefore, for the strong spin-orbit case, there appears a crossover phenomenon from the mean field behaviour with exponent $s=1$ to the critical behaviour with $s=0.57$ in three dimensions. Many materials are observed with exponent $s=1$ [13] and there may be exists such a behaviour among these materials.

\section{Acknowledgments.}

The author thanks Edouard Brézin warmly for useful discussions. He acknowledges the support of Yoshida Science Foundation and the hospitality of the Theoretical Physics Division at Saclay.

\section{References}

[1] Abrahams, E., Anderson, P. W., Licciardello, D. C. and Ramakrishnan, T. V., Phys. Rev. Lett. 42 (1979) 673.

[2] Wegner, F., Z. Phys. B 35 (1979) 207.

[3] Hikami, S., Prog. Theor. Phys. Suppl. (1985) « Anderson Localization » to be published.

[4] Efetov, K. B., Adv. Phys. 32 (1983) 53.

[5] Hikami, S., Larkin, A. J. and Nagaoka, Y., Prog. Theor. Phys. 63 (1980) 707.

[6] Hikami, S., Prog. Theor. Phys. 64 (1980) 1425.

[7] Vasil'ev, A. N., Nalimov, N. Yu. and Khonkonen, Yu. R., Theor. Math. Phys. 58 (1984) 111.

[8] BréZin, E., Hikami, S. and Zinn-Justin, J., Nucl. Phys. B 165 (1980) 528.

[9] Wegner, F. J., Nucl. Phys. B 180 (FS2) (1981) 174.

[10] Oppermann, R. and Jüngling, K., Phys. Lett. A 76 (1980) 449.

[11] Bergmann, G., Phys. Rep. 107 (1984) 1.

[12] Thomas, G. A., Ootuka, Y., Katsumoto, S., Kobayashi, S. and Sasaki, W., Phys. Rev. B 25 (1982) 4288.

[13] Thomas, G. A. and PaAlanen, M. A., Localization, Interaction and Transport Phenomena eds, B. Kramer, G. Bergmann and Y. Bruynseraede, Springer Series in Solid-State Sciences 61, (SpringerVerlag, Berlin) 1985. 